\title{
Iron Overload Adversely Effects Bone Marrow Haematogenesis via SIRT-SOD2-MROS in a Process Ameliorated by Curcumin
}

\section{shujuan zhou}

Wenzhou Medical University First Affiliated Hospital Department of Hematology https://orcid.org/0000-0003-3809-7613

\section{Lan Sun}

Wenzhou Medical University First Affiliated Hospital

\section{Shanhu Qian}

Wenzhou Medical University First Affiliated Hospital

\section{Yongyong $\mathrm{Ma}$}

Wenzhou Medical University First Affiliated Hospital Department of Hematology

\section{Ruye Ma}

Wenzhou Medical University First Affiliated Hospital Department of Hematology

\section{Yuqing Dong}

Wenzhou Medical University First Affiliated Hospital Department of Hematology

\section{Yifen Shi}

Wenzhou Medical University First Affiliated Hospital Department of Hematology

\section{Songfu Jiang}

Wenzhou Medical University First Affiliated Hospital Department of Hematology Haige Ye

Wenzhou Medical University First Affiliated Hospital Department of Hematology

\section{Zhijian Shen}

Wenzhou Medical University First Affiliated Hospital Department of Hematology

\section{Shenghui Zhang}

Wenzhou Medical University First Affiliated Hospital Department of Hematology

\section{Jianping Shen}

Zhejiang Chinese Medical University

\section{Kang Yu}

Wenzhou Medical University First Affiliated Hospital Department of Hematology

siqian wang ( $\nabla$ wsq98200@163.com )

The school and hospital of wenzhou medical university 
Keywords: curcumin, iron overload, bone marrow damage, autophagy, mitochondrial ROS

Posted Date: June 12th, 2020

DOI: https://doi.org/10.21203/rs.3.rs-33691/v1

License: (c) (1) This work is licensed under a Creative Commons Attribution 4.0 International License. Read Full License

Version of Record: A version of this preprint was published at Cellular \& Molecular Biology Letters on January 13th, 2021. See the published version at https://doi.org/10.1186/s11658-020-00244-7. 


\section{Abstract}

Background: Iron overload is common in patients with haematological disorders, and it is known to have a suppressive effect on haematogenesis. However, the mechanism by which iron overload affects haematogenesis is still unclear. The antioxidant curcumin has been reported to protect against iron overload-induced bone marrow damage, although the mechanism underlying this protective effect remains to be elucidated.

Methods: We established iron overload cell and mouse models. Mitochondrion-derived reactive oxygen species (mROS) levels, autophagy levels, and the SIRT3/SOD2 pathway were examined in these models and in the bone marrow of patients with iron overload.

Results: Iron overload was shown to depress haematogenesis and induce mitochondrion-derived superoxide anion-dependent autophagic cell death. Iron loading decreased SIRT3 protein expression, promoted an increase in SOD2, and led to the elevation of mROS. These effects were reversed by the overexpression of SIRT3. Curcumin treatment ameliorated peripheral blood cells, enhanced SIRT3 activity, decreased SOD2 acetylation, inhibited mROS production, and suppressed iron loading-induced autophagy.

Conclusions: These results suggest that curcumin exerts a protective effect on bone marrow by reducing mROS-stimulated autophagic cell death in a manner dependent on the SIRT3/SOD2 pathway.

\section{Introduction}

Iron overload is common in patients with haematological disorders due to long-term repeated red cell transfusion, congenital iron overload disease, and a subclass of anaemia characterized by ineffective haematogenesis, leading to excess iron deposition throughout the body. Such iron overload causes tissue damage and organ dysfunction and eventually leads to the mortality and morbidity associated with anaemia-related diseases ${ }^{1,2}$. There is accumulating clinical evidence that iron overload has a suppressive effect on haematogenesis and that iron chelation therapy can improve this condition ${ }^{3-7}$. The damaging effect of iron overload is due to the elevation in labile intracellular iron pools (LIP) in the cells that generate reactive oxygen species (ROS). However, the mechanism by which ROS damage haematopoietic cells remains unclear.

The mitochondrion is a focal point of iron metabolism. The mitochondrion is also a major source of ROS

${ }^{8}$. Mitochondrion-derived reactive oxygen species (mROS) have been shown to induce autophagy ${ }^{9-11}$. The molecular mechanism of autophagy has been widely investigated. Sirtuin 3 (SIRT3) is the major mitochondrial acetyl-lysine deacetylase that can modulate many proteins, such as superoxide dismutase 2 (SOD2), and then control mitochondrial function and mROS generation ${ }^{12}$. Moreover, SIRT3 can directly bind and deacetylate SOD2, which increases its activity and leads to a significant effect on mROS homeostasis and autophagic flux ${ }^{13,14}$. 
Curcumin is a naturally occurring yellow pigment isolated from ground rhizomes of the plant Curcuma

longa and has been shown to possess anti-inflammatory, antioxidant, and iron-chelating properties ${ }^{15,16}$. Given this complex array of interactions, curcumin represents a promising therapeutic option in the management of free radical-related diseases ${ }^{17}$. However, the ability of curcumin to protect against iron overload-induced autophagic cell death in human bone marrow remains unknown.

Previously, we found a deleterious impact of iron overload on haematogenesis. The present study was performed to examine whether curcumin supplementation could attenuate the haematogenesis abnormalities induced by iron overload and to explore the underlying mechanisms.

\section{Materials And Methods}

\section{Patients and healthy donors}

The study population consisted of six patients (three females and three males) diagnosed with thalassemia major. Six age-matched healthy donors were enrolled as controls. This study was carried out with approval from the Ethics Committee of the First Affiliated Hospital of Wenzhou Medical University, and written consent was obtained from all subjects prior to participation in this study, in accordance with the Declaration of Helsinki.

\section{Animal studies}

Sixty mice weighing 20-25 g were obtained from the Laboratory Animal Centre of Wenzhou Medical University. They were raised at the certified animal care facility of the First Affiliated Hospital of Wenzhou Medical University. The mice were randomized into an iron loading group $(200 \mathrm{mg} / \mathrm{kg}$ iron dextrin intraperitoneal injection, $1 \mathrm{ml} / \mathrm{kg}$ corn oil gavage, $n=15)$, a control group $(0.2 \mathrm{ml}$ normal saline intraperitoneal injection, $1 \mathrm{ml} / \mathrm{kg}$ corn oil gavage, $n=15)$, a curcumin group $(0.2 \mathrm{ml}$ normal saline intraperitoneal injection, $200 \mathrm{mg} / \mathrm{kg}$ curcumin gavage, $n=15)$, or a curcumin + iron loading group (200 mg/kg iron dextrin intraperitoneal injection, $200 \mathrm{mg} / \mathrm{kg}$ curcumin gavage, $n=15)$. The iron overload groups received an intraperitoneal injection of iron dextran every 3 days for 4 weeks. The curcumin groups were gavaged with $200 \mathrm{mg} / \mathrm{kg}$ curcumin every day for 4 weeks.

\section{Peripheral blood cell and bone marrow mononuclear cell (BMMNC) counts}

We obtained peripheral blood from mice via the orbital sinus and collected blood samples in ethylenediaminetetraacetic acid (EDTA) tubes. Complete blood counts were analysed by a pocH-100i haematology analyser (Sysmex, Kobe, Japan). The cell counts included red blood cells (RBCs), haemoglobin $(\mathrm{Hb})$, white blood cells (WBCs) and platelets (PLTs). The BMMNCs were flushed from the bones as described previously 18,19 and counted using the haematology analyser.

\section{Cell culture}


BMMNCs were isolated from bone marrow aspirates from healthy donors or patients with thalassemia major by Ficoll-Hypaque density gradient centrifugation, and immediately cultured in RPMI 1640 medium (Gibco, Grand Island, NY, USA) with 20\% fetal bovine serum (FBS; Gibco), which mainly consisted of progenitor and stem cells as determined by flow cytometric analyses of CD34 and CD38 expression. Cells were grown in a humidified atmosphere of $5 \% \mathrm{CO}_{2}$ at $37^{\circ} \mathrm{C}$. After 3 days, medium was changed. When cells reached confluence, they were replated at a density of $1 \times 10^{4} \mathrm{cells} / \mathrm{cm}^{2} .1 \times 10^{4}$ cells were cultured in RPMI 1640 medium containing $1.0 \%$ of methylcellulose, $30 \%$ of FBS, $1 \%$ of bovine serum albumin (BSA; Sigma-Aldrich) and $10^{-4} \mathrm{M}$ of 2-mercaptoethanol (Sigma-Aldrich) for following experiments.

\section{Cell experimental protocol}

The effects of ferric ammonium citrate (FAC) on autophagy in BMMNCs were evaluated. The cells were treated with FAC (F879; Sigma, St. Louis, MO) at different concentrations $(0,100,200,400 \mu \mathrm{M})$ for 24 hours. Next, we investigated the ability of curcumin (targetmol, T516) to alleviate FAC induced myelotoxicity. BMMNCs were pretreated with $30 \mu \mathrm{M}$ curcumin for 2 hours prior to FAC treatment. Finally, the role of the SIRT3-SOD2 pathway in mononuclear cell protection after curcumin pretreatment was investigated.

\section{Cell viability}

Cell viability was analysed using a Cell Counting Kit-8 according to the manufacturer's instructions (CK04; Dojindo Molecular Technologies, Kumamoto, Japan). The results are expressed as percentages relative to the control.

\section{Determination of mitochondrion-derived ROS}

To assess mROS, BMMNCs were incubated with culture medium containing $10 \mathrm{mM}$ MitoSOX (M36008; Invitrogen, Carlsbad, CA, USA) for 20 minutes at $37^{\circ} \mathrm{C}$. After incubation, fluorescence intensity was measured at an excitation wavelength of $492 \mathrm{~nm}$ and an emission wavelength of $595 \mathrm{~nm}$ using an Infinite ${ }^{\mathrm{TM}}$ M200 Microplate Reader (Tecan, Männedorf, Switzerland).

\section{Measurement of SOD2 enzyme activity}

SOD2 enzymatic activity was assayed using a SOD1 and SOD2 Assay Kit with WST-8 (S0103; Beyotime, Haimen, China) in accordance with the manufacturer's instructions. The $\mathrm{A}_{450}$ was measured using an Infinite ${ }^{\text {TM }}$ M200 Microplate Reader (Tecan).

\section{Plasmids and transfection}

The plasmid LV5-SIRT3 was designed by Yuxi Biotechnology Corp. (Yuxi, China). Mononuclear cells grown in Dulbecco's modified Eagle's medium (DMEM) with 20\% FBS for 24 hours were transfected with SIRT3 and control plasmids using Opti-MEM I-reduced serum media and Lipofectamine 2000 according to the manufacturer's instructions (11668-019; Invitrogen). Twenty-four hours after transfection, the cells were washed and processed for immunoblotting and other assays. 


\section{Real-time PCR analysis to detect SIRT3 mRNA}

All reagents used for real-time PCR were obtained from Life Technologies (Carlsbad, CA, USA). The SIRT3 probes were 5'-GACATTCGGGCTGACGTGAT-3' and 5'- ACCACATGCAGCAAGAACCTC-3'; the GAPDH probes were 5'-TGACAACAGCCTCAAGAT-3' and 5'-GAGTCCTTCCACGATACC-3'.

\section{SIRT3 activity}

SIRT3 enzymatic activity was assayed by following the manufacturer's instructions of a fluorometric kit (BML-AK557-0001; Enzo Life Sciences, Farmingdale, NY, USA).

\section{Western blotting analysis}

BMMNCs were washed twice and transferred to a new tube. Protein concentrations were determined. The protein samples were separated by SDS-PAGE. Following protein transfer onto polyvinylidene difluoride membranes, the membranes were blocked and then incubated overnight at $4{ }^{\circ} \mathrm{C}$ with antibodies against LC3, microtubule-associated protein 1 light chain 3( LC3) (1:1,000, L7543; Sigma), SIRT3 (1:100, sc99143; Santa Cruz Biotechnology, Santa Cruz, CA, USA), SOD2 (1:100, sc-33254; Santa Cruz Biotechnology), and $\beta$-actin (1:5,000, A5441; Sigma). The membranes were visualized by enhanced chemiluminescence using Super Signal West Pico blotting detection reagents (34079; Pierce, Rockford, IL, USA) and exposure to Hyper Performance Chemiluminescence film (Amersham, Little Chalfont, UK).

\section{Colony-forming cell assays}

To investigate the multipotency of haematopoietic progenitor cells, colony-forming units were assayed using methylcellulose culture medium (Stem Cell Technologies, Vancouver, BC, Canada). Aliquots of $1 \times$ $10^{5}$ cells were plated in 24-well plates and cultured for 14 days. Colony-forming unit erythroid (CFU-E), burst-forming unit erythroid (BFU-E), colony-forming unit granulocyte-macrophages (CFU-GM), and colony-forming unit Mix (CFU-Mix) were counted. The cells in each group were seeded in triplicate.

\section{Labile intracellular iron pool (LIP) analysis}

Cellular labile iron pool (LIP) level was assessed by Calcein-AM fluorescent Dye (Sigma) ${ }^{20}$. Briefly, aliquots of $3 \times 10^{6}$ cells were inoculated into 6-well plates. After treatment, the cells were washed twice with PBS and incubated with calcein-AM (CA-AM, $0.125 \mu \mathrm{mol} / \mathrm{L}$ ) for 10 minutes at $37^{\circ} \mathrm{C}$. After washing twice with PBS, the residue was combined with $0.25 \mu \mathrm{g} / \mathrm{ml}$ trypan blue solution and dispersed, and the fluorescence intensity was measured by fluorospectrophotometry with excitation and emission wavelengths of $495 \mathrm{~nm}$ and $530 \mathrm{~nm}$, respectively. Next, the samples were incubated with bipyridine (BIP) $(100 \mu \mathrm{M})$ for 30 minutes at $37^{\circ} \mathrm{C}$, and the fluorescence intensity was measured again under the same conditions. The difference in cellular fluorescence before versus after incubation with BIP reflects the amount of labile intracellular iron pools.

\section{Statistical analysis}


The data are presented as the mean \pm SEM and were analysed by $t$ test or one-way ANOVA. Data were analysed using GraphPad Prism- 5 software (GraphPad Software, San Diego, CA, USA). In all analyses, $P$ $<0.05$ was considered to indicate statistical significance.

\section{Results}

\section{Bone marrow damage caused by iron overload}

To determine whether iron overload could cause bone marrow damage, we established an iron overload cell model and found that iron-exposed BMMNCs showed decreased cell viability (Fig. S1-A), reduced cell proliferation activity (Fig. S1-B), and significantly increased intracellular labile intracellular iron pools (Fig. S1-C).

Then, an iron overload mouse model was established, and the results showed that the Hb, PLT count, and WBC count were significantly decreased in the peripheral blood (Fig. S3-A) and that mononuclear cell activity was decreased in the bone marrow of iron overloaded mice (Fig.S3-B). In addition, the labile intracellular iron pools in the mononuclear cells of iron overloaded mice were significantly higher than those in the normal control mice (Fig. S3-C).

We extracted bone marrow from iron overloaded patients with thalassemia and found decreased activity of BMMNCs (Fig. 7-A) and reduced proliferative activity of BMMNCs in the bone marrow of these patients (Fig. 7-B).

\section{Iron overload can lead to bone marrow damage through autophagy}

To clarify whether autophagy is involved in the BMMNC damage caused by iron overload, we first examined the expression of LC3 as a marker of autophagy in iron loaded BMMNCs. The results indicated that the expression of LC3II increased with increasing FAC dose (Fig. 1-A). Then, we examined ironexposed BMMNCs by confocal microscopy and found a significant increase in LC3-II expression and GFP-LC3-positive autophagosomes in the cells (Fig. 1-B).

A basal level of autophagy has a protective effect on cells, but excessive autophagy can induce cell death. To clarify the effects of autophagy caused by iron overload on BMMNCs, we examined the apoptosis of iron-exposed BMMNCs. The results revealed no obvious apoptosis of iron-exposed BMMNCs (data not shown). Next, iron-exposed BMMNCs were treated with bafilomycin A1 (BafA1), an inhibitor of the proton pump of autophagic lysosomes, which can inhibit autophagy and reduce the degradation of autophagosomes. Under treatment with BafA1, the concentration of LC3II increased (Fig. 1-C), suggesting that the increase in LC3Il under conditions of iron loading promoted the increase in autophagic flow. Cell viability was also improved by treatment with BafA1 (Fig. 1-D).

Iron overload increases autophagy through mROS 
Autophagy was reported to be related to $\mathrm{mROS}^{21}$. The initial oxygen reduction product in mitochondria is $\mathrm{O}_{2}$, which is then rapidly converted to $\mathrm{H}_{2} \mathrm{O}_{2}{ }^{22}$. Therefore, we examined the levels of mROS in ironexposed BMMNCs, and the results indicated significantly elevated mROS levels in these cells (Fig. 2-A). In animal experiments, we found that the mROS levels were significantly increased in mice under conditions of iron overload (Fig. S3-D). The levels of mROS were also found to be significantly higher in iron overloaded patients than in normal subjects (Fig. 7-D). BMMNCs were then pretreated with the mitochondrial antioxidant Mito-TEMPO, which targets SOD in mitochondria, for 2 hours before coculture with FAC. Mito-TEMPO inhibited the production of mROS (Fig. 2-B) and markedly inhibited the expression of LC3Il caused by iron loading (Fig. 2-C). Moreover, the viability of iron-exposed cells was significantly improved by pretreatment with Mito-TEMPO (Fig. 2-D). Taken together, these results suggested that mROS induced autophagy under iron loaded conditions, while antioxidants, such as Mito-TEMPO, can reduce oxidative stress in mitochondria and thus reduce autophagy.

\section{Iron overload can increase SOD2 acetylation by inhibiting SIRT3 activity and expression}

SOD2 is the main scavenger of oxygen free radicals in the mitochondria. It catalyses the conversion of $\mathrm{O}_{2} \cdot$ to $\mathrm{H}_{2} \mathrm{O}_{2}$ and plays an important role in regulating mROS in mitochondria. Therefore, we examined the effects of iron overload on SOD2 expression. Interestingly, the activity of SOD2 decreased with increasing FAC dose (Fig. 3-A), but no effect was observed on the levels of SOD2 protein expression (Fig. 3-B). SOD2 activity is regulated by the acetylation of lysine residues. We first detected the acetylation level of SOD2 by coimmunoprecipitation and Western blot analysis. The results indicated that iron exposure resulted in a progressive increase in the SOD2 acetylation level (Fig. 3-C). The deacetylation of SOD2 is mainly regulated by the mitochondrial deacetylase, SIRT3 ${ }^{6,7,23}$. Therefore, we examined SIRT3 activity and concentration in iron-exposed BMMNCs. Iron loading resulted in significant decreases in the levels of SIRT3 mRNA (Fig. 3-D) and protein (Fig. 3-B). This study also showed that the levels of SIRT3 activity in the iron loading group were significantly lower than in the control group (Fig. 3-E). We next performed iron overload mouse experiments and examined the bone marrow of iron overloaded patients, and the results verified that SOD2 activity (Fig. S3-E) and SIRT3 (Fig. S3-F) activity were significantly decreased under iron overload conditions. The activities of SOD2 (Fig. 7-E) and SIRT3 (Fig. 7-F) in BMMNCs from patients with iron overload were also significantly decreased.

\section{SIRT3-SOD2 modulates iron overload-induced mROS accumulation and autophagy}

To examine whether the recovery of SIRT3 protein level or activity could prevent autophagy due to iron loading, we transfected SIRT3 via Lipofectamine 2000 into BMMNCs, resulting in its overexpression. The results indicated that overexpression of SIRT3 ameliorated the inhibition of SIRT3 activity induced by iron loading (data not shown), reduced the acetylation of SOD2 (Fig. 4-A) and increased the activity of SOD2 (Fig. 4-B), effectively reducing the mROS produced by iron loading (Fig. 4-C). SIRT3 overexpression 
increased cell viability and significantly reduced LC3II expression in iron-exposed cells (Fig. $<$ link rid="fig5">4</link>-E and 4-D, respectively).

\section{Curcumin protects against iron overload-induced autophagic cell death}

Next, we examined whether curcumin, which has an antioxidative effect ${ }^{24}$, could protect cells against the oxidative stress and autophagy caused by iron loading. The results indicated that curcumin reduced the elevation of mROS induced by iron exposure (Fig. S2-A), while there was no significant decrease in intracellular labile iron pools (Fig. S2-B). Curcumin partially recovered cell viability (Fig. S2-C) and reduced the expression of LC3II (Fig. S2-D). In the mouse experiments, we also found that cell viability was markedly better in the iron loading + curcumin group than in the iron loading group (Fig. 6-A); furthermore, the labile intracellular iron pools in the cells were similar (Fig. 6-B), the LC3Il level was significantly decreased (Fig. 6-C), and the haemoglobin, platelet count, and white blood cell count were elevated (Fig. 6-D) (Table-1).

Table 1

Peripheral blood cell indices of mice.

\begin{tabular}{|llll|}
\hline & White blood cell $\left(\times 10^{9} / \mathrm{L}\right)$ & hemoglobin $(\mathrm{g} / \mathrm{L})$ & Platelets $\left(\times 10^{11} / \mathrm{L}\right)$ \\
\hline Control & $15.2 \pm 5.2$ & $18.4 \pm 1.3$ & $9.3 \pm 2.1$ \\
\hline Cur & $14.1 \pm 4.8$ & $17.3 \pm 0.8$ & $9.7 \pm 1.5$ \\
\hline CuC & $4.0 \pm 1.2^{*}$ & $14.9 \pm 0.7^{*}$ & $6.3 \pm 1.0^{*}$ \\
\hline * $\mathrm{FAC}<0.05$, versus the control group, ${ }^{*} \mathrm{p}<0.05$ vs. the FAC group. $(\mathrm{n}=15)$ \\
\hline WBC, white blood cell; HB, hemoglobin; PLT, platelet.
\end{tabular}

\section{Curcumin inhibits iron overload-induced autophagy through a SIRT3-SOD2-dependent mechanism}

SIRT3 is related to the autophagy and bone marrow damage caused by iron overload. Therefore, we examined the relationship between curcumin and SIRT3. Interestingly, curcumin partially restored the inhibition of SIRT3 activity induced by iron loading (Fig. 5-D) and the SIRT3 protein level (Fig. 5-B). As expected, curcumin pretreatment reduced mitochondrial-derived ROS production (Fig. 5-A), and inhibited the SOD2 acetylation (Fig. 5-C) induced by iron loading and restored SOD2 activity, but it had a minimal effect on the level of SOD2 protein (Fig. 5-B). In animal experiments, the SIRT3 activity of the iron loading + curcumin group was significantly higher than that of the iron loading group (Fig. 6-F), and SOD2 activity was restored (Fig. 6-E). 


\section{Discussion}

Iron overload is a common complication in haematological patients that can lead to a significant deficit in haematogenesis due to ROS-related injury. Curcumin supplementation decreases the oxidative stress produced by chronic iron intake and improves the antioxidant defence mechanism. Our experiments indicated that iron overload leads to the dysfunction of SIRT3-SOD2 in the mitochondria, resulting in the overproduction of mROS. Curcumin reduces the production of mROS through the SIRT3-SOD2 signalling pathway and decreases the autophagy level of cells. Importantly, curcumin shows a compensatory and protective role in eliminating mROS and suppressing autophagy through the SIRT3-SOD2 pathway both in vitro and in vivo and restores the activity of BMMNCs.

Numerous reports have demonstrated that iron overload has negative effects on haematogenesis ${ }^{25,26}$. Iron chelation was shown to lead to decreased transfusion requirements and increased platelet and neutrophil counts, thus providing indirect evidence for the toxic effect of iron overload on haematogenesis ${ }^{25-27}$. Furthermore, haematopoietic progenitor cells also showed suppressed colonyforming capacity under conditions of iron overload ${ }^{27,28}$. The present study indicated decreased viability of iron-exposed BMMNCs and decreased cell proliferation activity both in vitro and in vivo, but no obvious apoptosis was observed. The haemoglobin, platelet count, and white blood cell count in peripheral blood were markedly decreased in iron overloaded mice.

Many studies have indicated that iron overload leads to increased ROS levels. Haematopoietic stem cells are predominantly present in the low oxygen milieu of bone marrow, and high levels of ROS are harmful to normal haematogenesis ${ }^{29,30}$. However, ROS are also known to induce autophagy. Appropriate levels of autophagy are necessary to maintain homeostasis, but excessive levels of autophagy promote cell death ${ }^{14}$. There have been few reports addressing whether autophagy occurs and its significance in iron overload. In the present study, we showed that iron overload disrupted normal haematogenesis and led to increased mROS levels. These observations were consistent with the results of other in vitro studies ${ }^{31-33}$.

Although mitochondrial oxidative stress is associated with autophagy, the molecular mechanism underlying the accumulation of oxygen free radicals from the mitochondria remains unclear. Cells rigorously regulate the oxygen free radicals derived from mitochondria. Mitochondria have specific mechanisms for removing excess oxygen free radicals to maintain homeostasis. SOD2 is an enzyme involved in scavenging free radicals and is only present in mitochondria ${ }^{34,35}$. As mitochondria consume $90 \%$ of intracellular oxygen, SOD2 activity is very important to maintain the balance of oxygen free radicals derived from mitochondria ${ }^{36,37}$. The amount of SOD2 protein is regulated at the level of transcription, and its activity is regulated by acetylation ${ }^{36-38}$. Consistent with previous studies, iron overload resulted in an elevated level of SOD2 acetylation in a dose-dependent manner in the present study. These data suggest that iron overload increases mitochondrial oxygen free radicals by increasing SOD2 acetylation levels, rather than by decreasing SOD2 protein expression. 
Lysine acetylation is an important posttranslational event in the regulation of mitochondrial proteins and autophagy ${ }^{39-41}$. SIRT3 is the strongest deacetylase in mitochondria and is directly involved in mitochondrial energy synthesis and in the control of oxygen free radical levels ${ }^{42}$. SIRT3 regulates SOD2 activity by regulating the acetylation level of SOD2, and its target lysine has been identified. SIRT3 deacetylates SOD2 in response to ionizing radiation, indicating that SOD2 is a major downstream signal of SIRT3-mediated mitochondrion-derived $\mathrm{O}_{2}$ reduction ${ }^{38}$. The results of the present study indicated that iron overload did not lead to a decrease in SOD2 expression but instead to increased acetylation levels, and we found decreases in both SIRT3 expression and activity. SIRT3 overexpression was shown to ameliorate the increase in SOD2 acetylation caused by iron overload. These studies indicated that SIRT3-SOD2-mediated autophagy is an important mechanism of the bone marrow injury induced by iron overload.

Curcumin is a natural antioxidant compound extracted from the rhizome of Zingiberaceae plants that has a variety of biological functions. Recent studies 43,44 have shown that curcumin can significantly alleviate the oxidative damage caused by stress through mitochondrial subunit localization. Waseem et al. reported that curcumin significantly alleviated cisplatin-induced oxidative damage in liver and brain tissues via the mitochondrial pathway ${ }^{45}$. Our results showed that curcumin can restore the activity of SIRT3, reduce the level of SOD2 acetylation, and restore the activity of SOD2, thereby reducing the production of mROS.

\section{Conclusion}

In summary, we propose a possible mechanism whereby iron overload induced bone marrow damage via mROS dependent autophagy. And curcumin shows a protective role in eliminating mROS and suppressing autophagy through the SIRT3-SOD2 pathway both in vitro and in vivo. Taken together, these findings provide new insights into the link between curcumin and autophagy signaling, which could contribute to a better understanding of protective effect in iron overload-induced bone marrow damage.

\section{Declarations}

\section{Ethics approval and consent to participate:}

This study was carried out with approval from the Ethics Committee of the First Affiliated Hospital of Wenzhou Medical University, and written consent was obtained from all subjects prior to participation in this study, in accordance with the Declaration of Helsinki.

\section{Consent for publication:}

Not applicable.

\section{Availability of data and materials:}


Not applicable.

\section{Competing interests:}

The authors declare that they have no competing interests.

\section{Funding}

This work was funded by the National Natural Science Foundation of China (No. 81703851).

\section{Author contribution:}

Kang Yu, Jianping Shen, Siqian Wang designed research. Shujuan Zhou, Siqian Wang, Lan Sun performed research. Shanhu Qian, Yongyong Ma, Ruye Ma, Yuqing Dong contributed vital new reagents or analytical tools. Songfu Jiang, Haige Ye collected data. Yifen Shi, Zhijian Shen and shenghui zhang analyzed and interpreted data. Siqian Wang performed statistical analysis. Shujuan Zhou, Siqian Wang wrote the manuscript.

\section{Conflict of interest!}

The author(s) declared no potential conflicts of interest with respect to the research, authorship, and/or publication of this article.

\section{Acknowledgements:}

This work was funded by the National Natural Science Foundation of China (No. 81703851).

\section{Abbreviations}

mROS, Mitochondrion-derived reactive oxygen species

LIP, labile intracellular iron pools

LC3, microtubule-associated protein 1 light chain 3

ROS, reactive oxygen species

SIRT3, Sirtuin 3

SOD2, superoxide dismutase 2

BMMNC, bone marrow mononuclear cell

EDTA, ethylenediaminetetraacetic acid 
WBCs, white blood cells

$\mathrm{Hb}$, haemoglobin

RBCs, red blood cells

PLTs, platelets

FBS, fetal bovine serum

BSA, bovine serum albumin

FAC, ferric ammonium citrate

CFU-E, Colony-forming unit erythroid

BFU-E, burst-forming unit erythroid

CFU-GM, colony-forming unit granulocyte-macrophages

CFU-Mix, colony-forming unit Mix

BIP, bipyridine

BafA1, bafilomycin A1

\section{References}

1. Kim IH, Moon JH, Lim SN, et al. Efficacy and safety of deferasirox estimated by serum ferritin and labile plasma iron levels in patients with aplastic anemia, myelodysplastic syndrome, or acute myeloid leukemia with transfusional iron overload. Transfusion (Paris). Jul 2015;55(7):1613-1620.

2. Pawelec K, Salamonowicz M, Panasiuk A, et al. Influence of Iron Overload on Immunosuppressive Therapy in Children with Severe Aplastic Anemia. Adv. Exp. Med. Biol. 2015;866:83-89.

3. Oliva EN, Ronco F, Marino A, Alati C, Pratico G, Nobile F. Iron chelation therapy associated with improvement of hematopoiesis in transfusion-dependent patients. Transfusion (Paris). Jul 2010;50(7):1568-1570.

4. Heli $\mathrm{H}$, Mirtorabi S, Karimian K. Advances in iron chelation: an update. Expert Opin. Ther. Pat. Jun 2011;21(6):819-856.

5. List AF, Baer MR, Steensma DP, et al. Deferasirox reduces serum ferritin and labile plasma iron in RBC transfusion-dependent patients with myelodysplastic syndrome. J. Clin. Oncol. Jun 10 2012;30(17):2134-2139.

6. Messa E, Cilloni D, Messa F, Arruga F, Roetto A, Saglio G. Deferasirox treatment improved the hemoglobin level and decreased transfusion requirements in four patients with the myelodysplastic 
syndrome and primary myelofibrosis. Acta Haematol. 2008;120(2):70-74.

7. Angelucci E, Santini V, Di Tucci AA, et al. Deferasirox for transfusion-dependent patients with myelodysplastic syndromes: safety, efficacy, and beyond (GIMEMA MDS0306 Trial). Eur. J. Haematol. Jun 2014;92(6):527-536.

8. Stowe DF, Camara AK. Mitochondrial reactive oxygen species production in excitable cells: modulators of mitochondrial and cell function. Antioxid Redox Signal. Jun 2009;11(6):1373-1414.

9. Scherz-Shouval R, Shvets E, Elazar Z. Oxidation as a post-translational modification that regulates autophagy. Autophagy. Jul-Aug 2007;3(4):371-373.

10. Filomeni G, De Zio D, Cecconi F. Oxidative stress and autophagy: the clash between damage and metabolic needs. Cell Death Differ. Mar 2015;22(3):377-388.

11. Mi Y, Xiao C, Du Q, Wu W, Qi G, Liu X. Momordin Ic couples apoptosis with autophagy in human hepatoblastoma cancer cells by reactive oxygen species (ROS)-mediated PI3K/Akt and MAPK signaling pathways. Free Radic. Biol. Med. Jan 2016;90:230-242.

12. Bause AS, Haigis MC. SIRT3 regulation of mitochondrial oxidative stress. Exp. Gerontol. Jul 2013;48(7):634-639.

13. Qiu X, Brown K, Hirschey MD, Verdin E, Chen D. Calorie restriction reduces oxidative stress by SIRT3mediated SOD2 activation. Cell Metab. Dec 1 2010;12(6):662-667.

14. Liang Q, Benavides GA, Vassilopoulos A, Gius D, Darley-Usmar V, Zhang J. Bioenergetic and autophagic control by Sirt3 in response to nutrient deprivation in mouse embryonic fibroblasts. Biochem. J. Sep 1 2013;454(2):249-257.

15. Zhang J, Hou X, Ahmad H, Zhang H, Zhang L, Wang T. Assessment of free radicals scavenging activity of seven natural pigments and protective effects in AAPH-challenged chicken erythrocytes. Food Chem. Feb 15 2014;145:57-65.

16. Messner DJ, Sivam G, Kowdley KV. Curcumin reduces the toxic effects of iron loading in rat liver epithelial cells. Liver Int. Jan 2009;29(1):63-72.

17. Calabrese $V$, Bates TE, Mancuso $C$, et al. Curcumin and the cellular stress response in free radicalrelated diseases. Mol. Nutr. Food Res. Sep 2008;52(9):1062-1073.

18. Meng A, Wang Y, Brown SA, Van Zant G, Zhou D. lonizing radiation and busulfan inhibit murine bone marrow cell hematopoietic function via apoptosis-dependent and -independent mechanisms. Exp. Hematol. Dec 2003;31(12):1348-1356.

19. Zhang $\mathrm{H}$, Zhai Z, Wang $\mathrm{Y}$, et al. Resveratrol ameliorates ionizing irradiation-induced long-term hematopoietic stem cell injury in mice. Free Radic. Biol. Med. Jan 2013;54:40-50.

20. Epsztejn S, Kakhlon O, Glickstein H, Breuer W, Cabantchik I. Fluorescence analysis of the labile iron pool of mammalian cells. Anal Biochem. May 15 1997;248(1):31-40.

21. Sena LA, Chandel NS. Physiological roles of mitochondrial reactive oxygen species. Mol. Cell. Oct 26 2012;48(2):158-167. 
22. Lambert AJ, Brand MD. Reactive oxygen species production by mitochondria. Methods Mol. Biol. 2009;554:165-181.

23. Di Tucci AA, Murru R, Alberti D, Rabault B, Deplano S, Angelucci E. Correction of anemia in a transfusion-dependent patient with primary myelofibrosis receiving iron chelation therapy with deferasirox (Exjade, ICL670). Eur. J. Haematol. Jun 2007;78(6):540-542.

24. Han J, Pan XY, Xu Y, et al. Curcumin induces autophagy to protect vascular endothelial cell survival from oxidative stress damage. Autophagy. May 1 2012;8(5):812-825.

25. Jensen PD, Heickendorff L, Pedersen B, et al. The effect of iron chelation on haemopoiesis in MDS patients with transfusional iron overload. Br. J. Haematol. Aug 1996;94(2):288-299.

26. Gattermann N, Finelli C, Della Porta M, et al. Hematologic responses to deferasirox therapy in transfusion-dependent patients with myelodysplastic syndromes. Haematologica. Sep 2012;97(9):1364-1371.

27. Saigo K, Takenokuchi M, Hiramatsu Y, et al. Oxidative stress levels in myelodysplastic syndrome patients: their relationship to serum ferritin and haemoglobin values. J. Int. Med. Res. 2011;39(5):1941-1945.

28. Juvonen E, Sahlstedt L, Parkkinen J, Ruutu T. Inhibition of erythroid and granulocyte-macrophage colony formation by non-transferrin-bound iron in vitro: protective effect of apotransferrin. Eur. J. Haematol. Aug 2007;79(2):126-131.

29. Jang YY, Sharkis SJ. A low level of reactive oxygen species selects for primitive hematopoietic stem cells that may reside in the low-oxygenic niche. Blood. Oct 15 2007;110(8):3056-3063.

30. Urao N, Ushio-Fukai M. Redox regulation of stem/progenitor cells and bone marrow niche. Free Radic. Biol. Med. Jan 2013;54:26-39.

31. Lu W, Zhao M, Rajbhandary S, et al. Free iron catalyzes oxidative damage to hematopoietic cells/mesenchymal stem cells in vitro and suppresses hematopoiesis in iron overload patients. Eur. J. Haematol. Sep 2013;91(3):249-261.

32. Huang H, Chen J, Lu H, Zhou M, Chai Z, Hu Y. Iron-induced generation of mitochondrial ROS depends on AMPK activity. Biometals. Aug 2017;30(4):623-628.

33. Yang F, Yan G, Li Y, et al. Astragalus Polysaccharide Attenuated Iron Overload-Induced Dysfunction of Mesenchymal Stem Cells via Suppressing Mitochondrial ROS. Cell. Physiol. Biochem. 2016;39(4):1369-1379.

34. Zeng L, Yang Y, Hu Y, et al. Age-related decrease in the mitochondrial sirtuin deacetylase Sirt3 expression associated with ROS accumulation in the auditory cortex of the mimetic aging rat model. PLoS One. 2014;9(2):e88019.

35. Li M, Chiu JF, Mossman BT, Fukagawa NK. Down-regulation of manganese-superoxide dismutase through phosphorylation of FOXO3a by Akt in explanted vascular smooth muscle cells from old rats. J. Biol. Chem. Dec 29 2006;281(52):40429-40439.

36. Chen Y, Zhang J, Lin Y, et al. Tumour suppressor SIRT3 deacetylates and activates manganese superoxide dismutase to scavenge ROS. EMBO Rep. Jun 2011;12(6):534-541. 
37. Zhu Y, Park SH, Ozden O, et al. Exploring the electrostatic repulsion model in the role of Sirt3 in directing MnSOD acetylation status and enzymatic activity. Free Radic. Biol. Med. Aug 15 2012;53(4):828-833.

38. Tao R, Coleman MC, Pennington JD, et al. Sirt3-mediated deacetylation of evolutionarily conserved lysine 122 regulates MnSOD activity in response to stress. Mol. Cell. Dec 22 2010;40(6):893-904.

39. Banreti A, Sass M, Graba Y. The emerging role of acetylation in the regulation of autophagy. Autophagy. Jun 1 2013;9(6):819-829.

40. Papanicolaou KN, O'Rourke B, Foster DB. Metabolism leaves its mark on the powerhouse: recent progress in post-translational modifications of lysine in mitochondria. Front. Physiol. 2014;5:301.

41. Hebert AS, Dittenhafer-Reed KE, Yu W, et al. Calorie restriction and SIRT3 trigger global reprogramming of the mitochondrial protein acetylome. Mol. Cell. Jan 10 2013;49(1):186-199.

42. Giralt A, Villarroya F. SIRT3, a pivotal actor in mitochondrial functions: metabolism, cell death and aging. Biochem. J. May 15 2012;444(1):1-10.

43. Zhang M, Tang J, Li Y, et al. Curcumin attenuates skeletal muscle mitochondrial impairment in COPD rats: PGC-1alpha/SIRT3 pathway involved. Chem. Biol. Interact. Nov 1 2017;277:168-175.

44. Naserzadeh P, Mehr SN, Sadabadi Z, Seydi E, Salimi A, Pourahmad J. Curcumin Protects Mitochondria and Cardiomyocytes from Oxidative Damage and Apoptosis Induced by Hemiscorpius Lepturus Venom. Drug Res (Stuttg). Feb 2018;68(2):113-120.

45. Waseem M, Parvez S. Mitochondrial dysfunction mediated cisplatin induced toxicity: modulatory role of curcumin. Food Chem Toxico/Mar 2013;53:334-42.

\section{Figures}

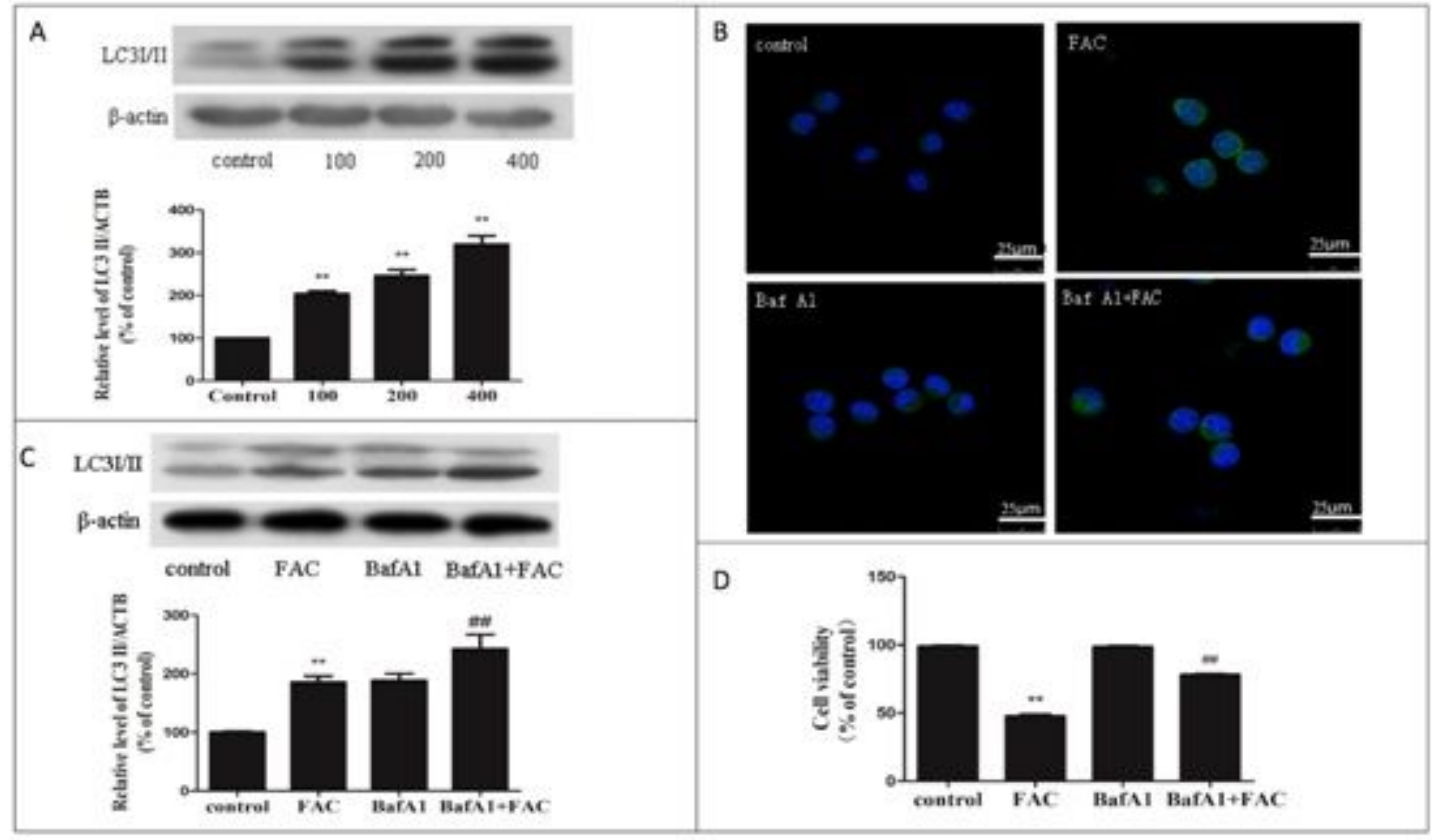


Iron overload can induces autophagic cell death in bone marrow mononuclear cells in vitro. A. A representative immunoblot analysis of LC3 assayed after mononuclear cells were treated with FAC at different concentrations $(0,100,200,400 \mu \mathrm{M})$ for $24 \mathrm{~h}$. $\beta$-actin used as an internal standard for protein loading(Repeat three times). B. Electron microscopy revealed an increased number of autophagic vacuoles when cells were treated with FAC at different concentrations $(0,100,200,400 \mu \mathrm{M})$ for $24 \mathrm{~h}$. Bone marrow mononuclear cells were incubated with FAC $(200 \mu \mathrm{M})$ in the absence or presence of BafA1 $(10 \mathrm{nM})$ for $24 \mathrm{~h}$ (Repeat three times) (C). The cell viability were recovered in the presence of Baf A1 $(10 \mathrm{nM})$ for $24 \mathrm{~h}(\mathrm{D})$. The values are presented as the means $\pm S E M,{ }^{\star *} \mathrm{p}<0.05$, versus the control group, $\# \# p<0.05$ vs. the FAC group. $\llbracket n=6 \rrbracket$

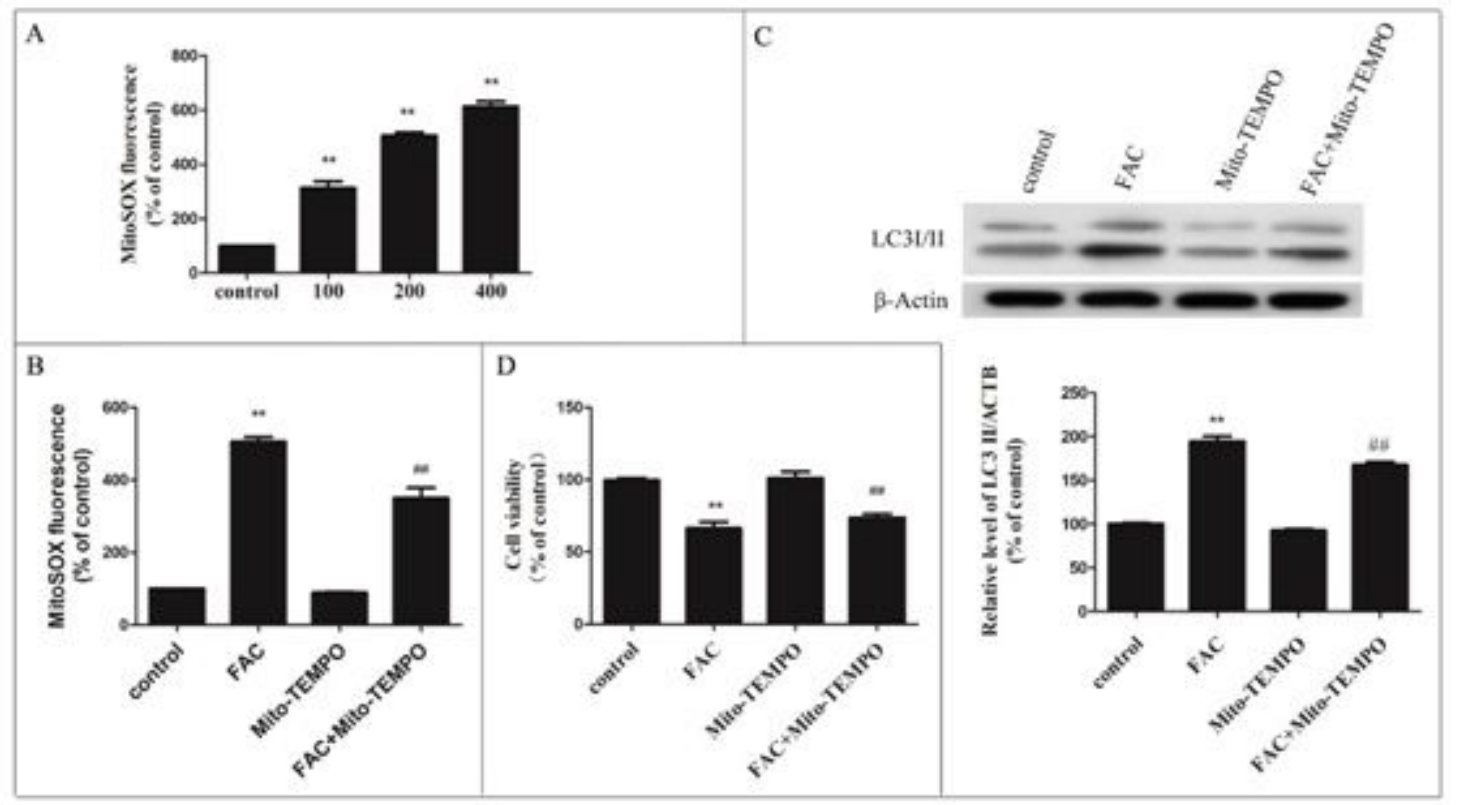

Figure 2

Mitochondrial ROS mediates iron overload induced autophagy in bone marrow mononuclear cells. A. Quantification of mitochondrial derived ROS levels using a fluorescence spectrometer after bone marrow mononuclear cells were treated with FAC at different concentrations for $24 \mathrm{~h}$. Bone marrow mononuclear cells were preincubated with Mito-TEMPO $(10 \mathrm{mM})$ for $2 \mathrm{~h}$ and then treated with $200 \mu \mathrm{M} \mathrm{FAC}$, then the mitochondrial derived ROS levels (B), LC3 level (C) and cell viability (D) were determined. The values are presented as the means $\pm S E M, * * p<0.05$, versus the control group, \#\#p<0.05 vs. the FAC group. 


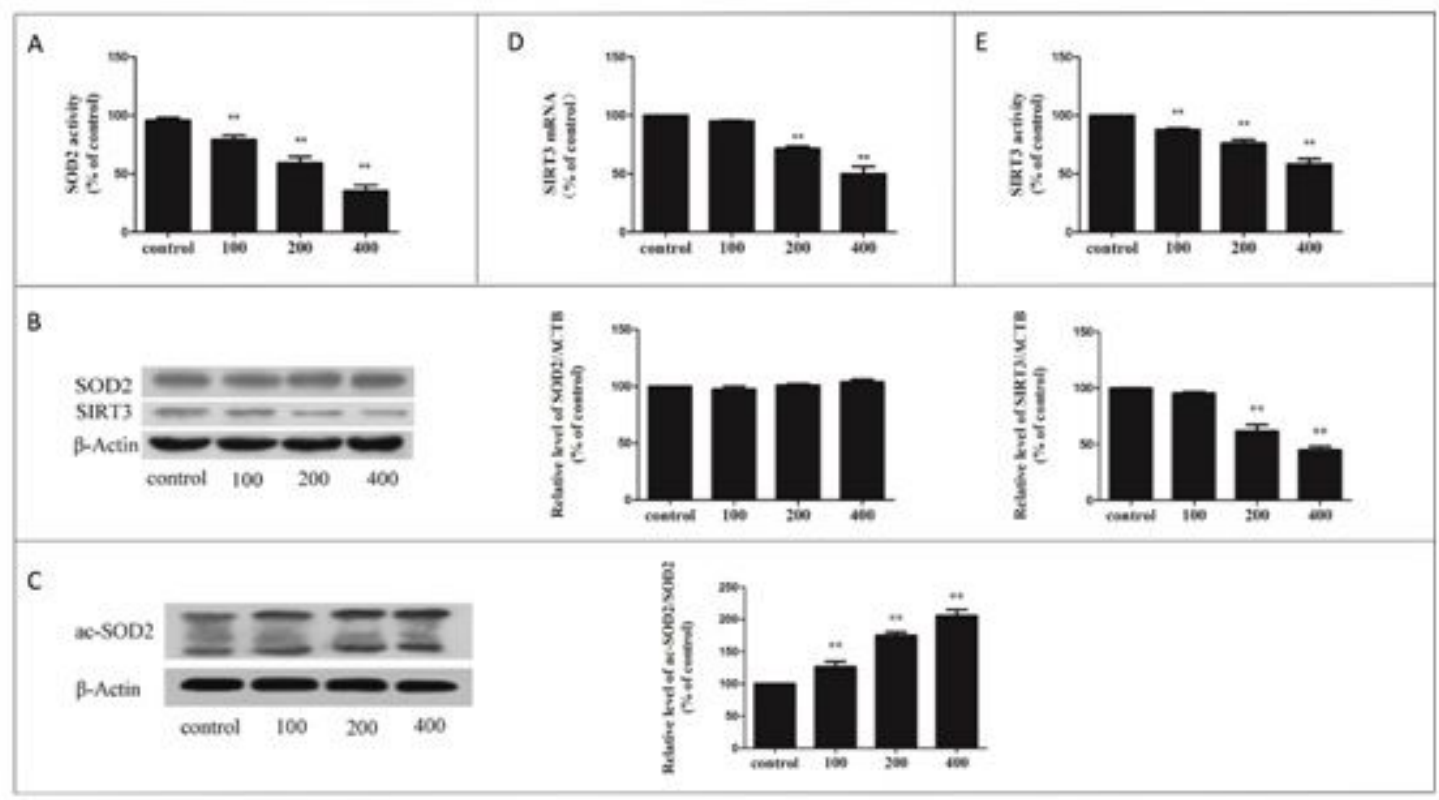

Figure 3

Iron overload can increase acetylated-SOD2 expression and decrease SIRT3 protein expression and activity in a dose-dependent manner. A. SOD2 activity in bone marrow mononuclear cells treated with FAC $(\mu \mathrm{M})$. B. Representative immunoblot of SOD2 and SIRT3 protein levels in bone marrow mononuclear cells, $\beta$-actin used as an internal standard for protein loading. C. Acetylation of SOD2 after FAC exposure was determined by immunoprecipitation with an anti-SOD2 antibody, followed by immunoblot analysis of acetylated-lysine, $\beta$-actin used as an internal standard for protein loading. D. Quantitative real-time PCR analysis was applied to detect SIRT3 mRNA levels. E. SIRT3 activity was measured based on an enzymatic reaction using a SIRT3 assay kit. The values are presented as the means $\pm S E M, * * p<0.05$, versus the control group.

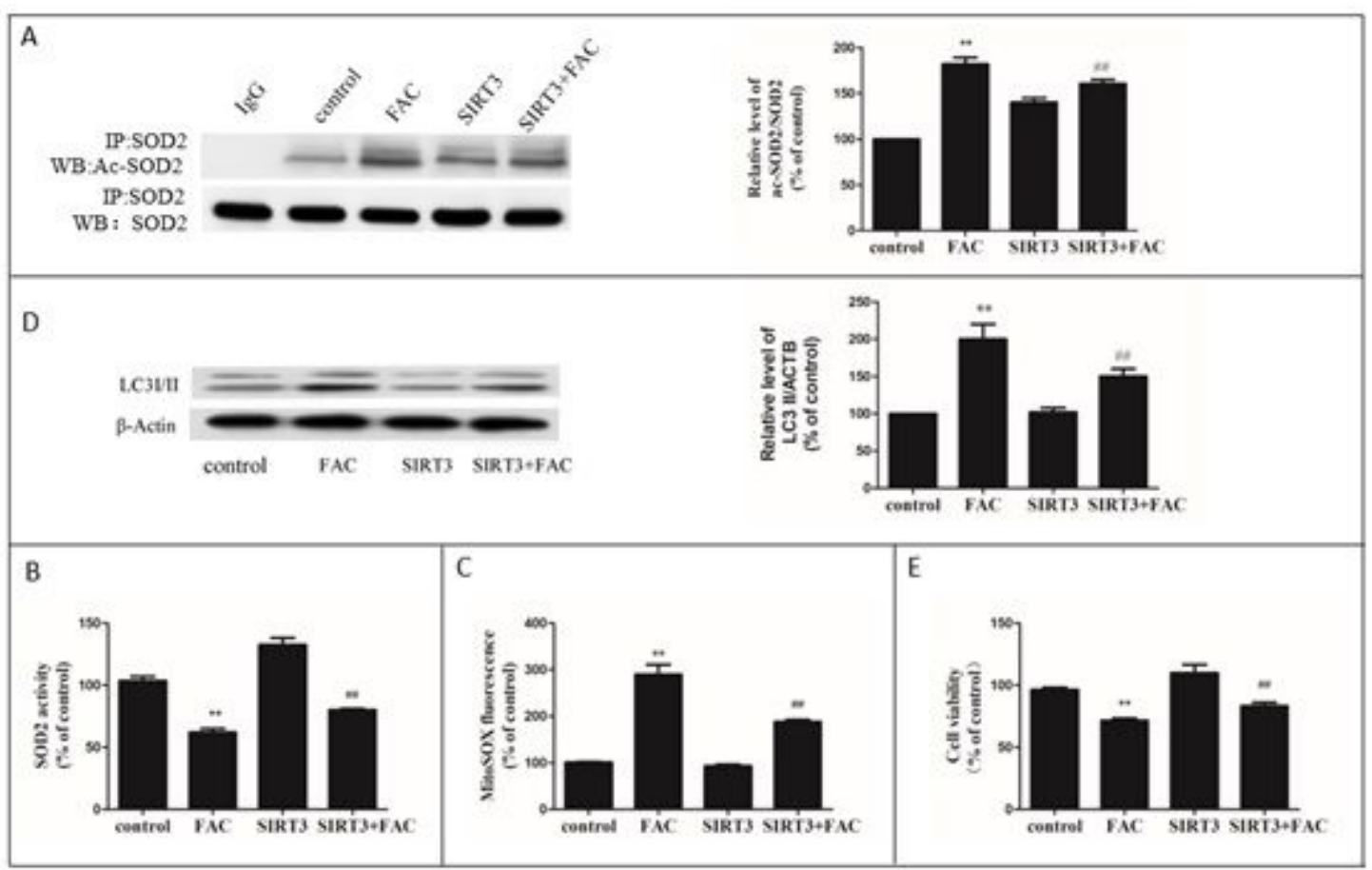


Figure 4

SIRT3-SOD2 modulates iron overload induced mROS accumulation and autophagy in bone marrow mononuclear cells in vitro. A. SIRT3 overexpression induced deacetylation of SOD2 (ac-SOD2) after $200 \mu$ M FAC treatment. After SIRT3 overexpression, SOD2 activity in bone marrow mononuclear cells (B), mitochondrial ROS production in bone marrow mononuclear cells $(C)$ and cell viability $(E)$ were tested. Representative immunoblot of LC3 protein levels in bone marrow mononuclear cells, $\beta$-actin used as an internal standard for protein loading(D). The values are presented as the means $\pm S E M,{ }^{* \star} p<0.05$, versus the control group, \#\#p<0.05 vs. the FAC group.

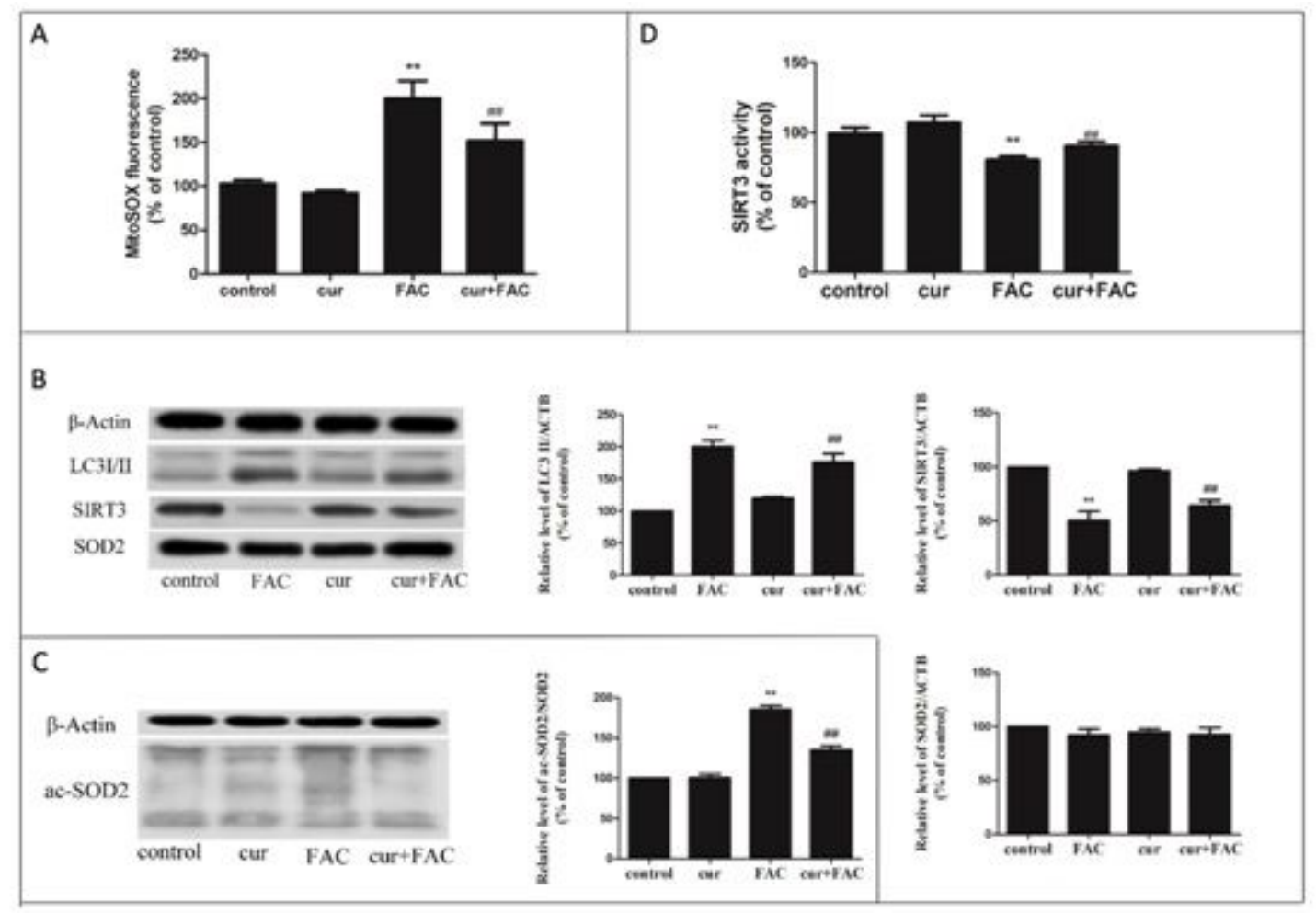

Figure 5

Curcumin pretreatment reduces iron overload induced autophagy in bone marrow mononuclear cells in vitro. A. The effects of curcumin pretreatment on mitochondrial-derived ROS production. A representative immunoblot analysis of SIRT3 (B) SOD2 protein(B) and SOD2 acetylation (C). The effects of curcumin pretreatment on SIRT3 activity (D). The values are presented as the means $\pm S E M,{ }^{* *} p<0.05$, versus the control group, \#\#p<0.05 vs. the FAC group. 


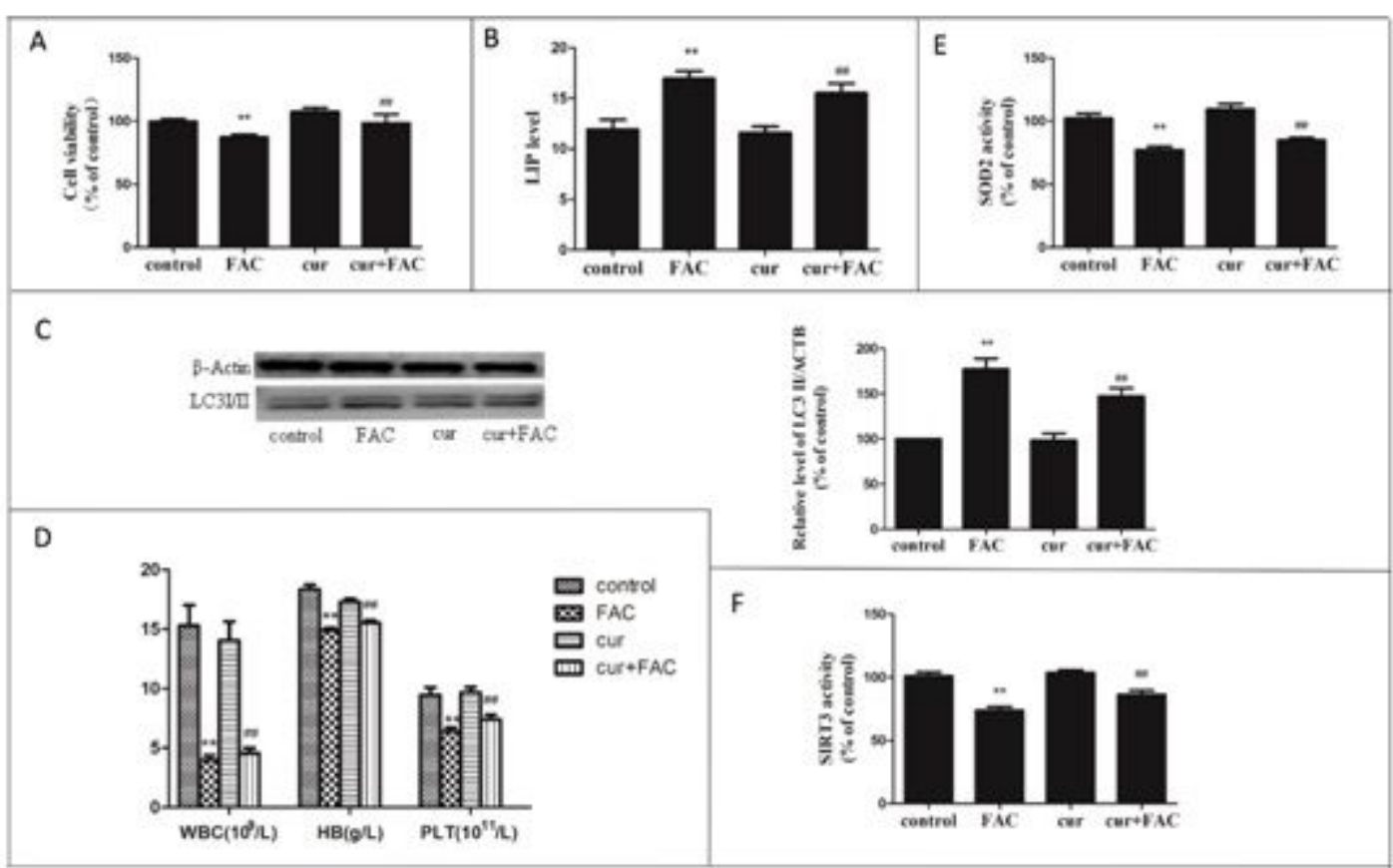

Figure 6

Curcumin protects against iron overload induced autophagic cell death in vivo $(n=15)$. A. Cell viability. B. The LIP in the cells. C. A representative immunoblot analysis of LC3.D. Peripheral blood test. SOD2(E) and SIRT3(F) activity in mouse treated by curcumin. The results are expressed as a percentage of the control group, which is set at $100 \%$. The values are presented as the means $\pm S E M, * \star p<0.05$, versus the control group, \#\#p<0.05 vs. the FAC group. 


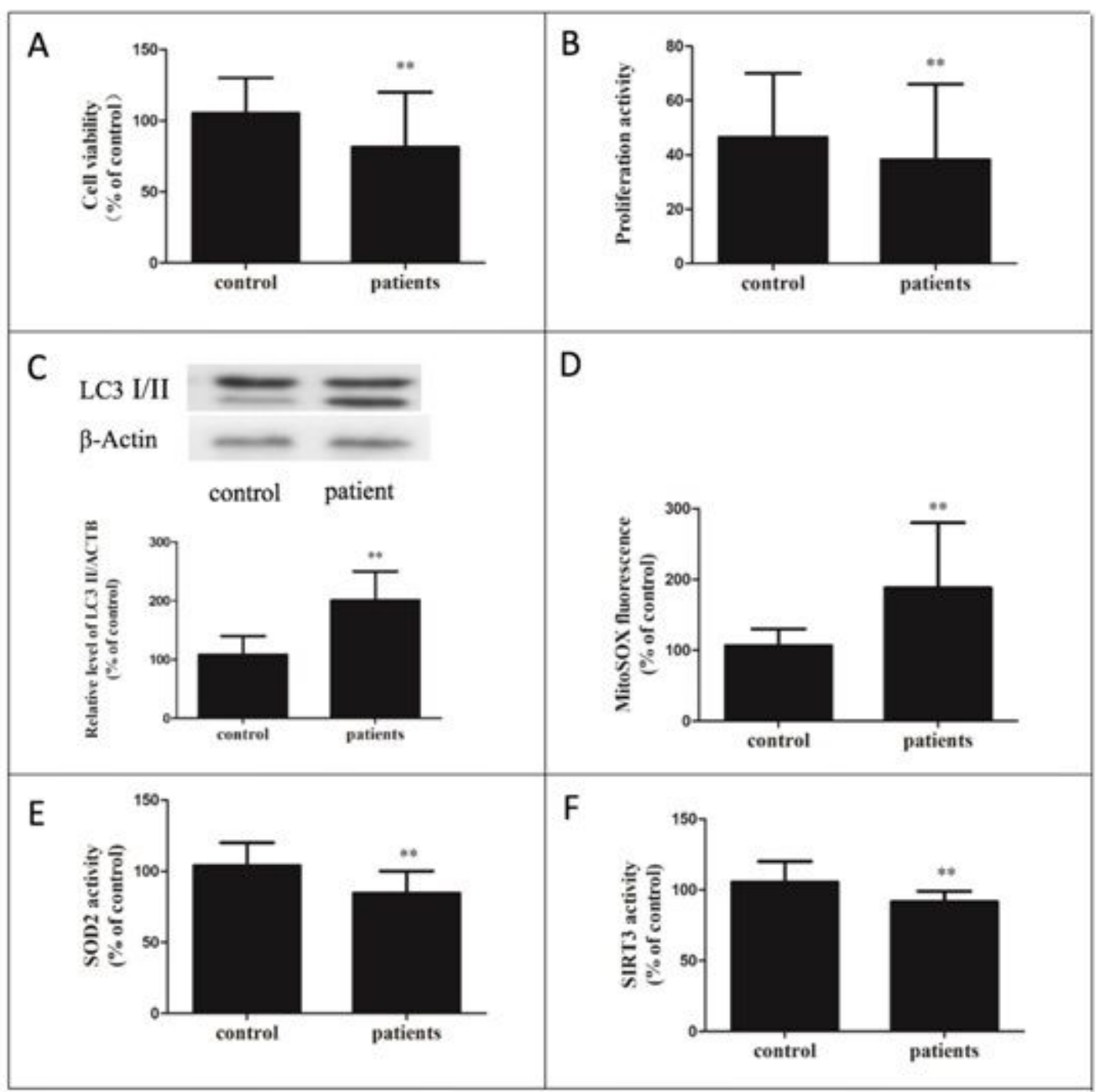

Figure 7

Iron overload affects the patients' bone marrow mononuclear cells. The cell viability $(A)$ and proliferation activity (B) of mononuclear cells in the bone marrow of the iron overload patients was decreased.LC3 levels (C) and mitochondrial-derived ROS levels (D) was increased, and SOD2 (E) and SIRT3 (F) activity was decreased in iron overload patients $(n=6)$. The results are expressed as a percentage of the control group, which is set at $100 \%$. The values are presented as the means $\pm S E M, * \star p<0.05$, versus the control group. 